\title{
Cola beverage consumption delays alveolar bone healing: a histometric study in rats
}

\section{Juliana Mazzonetto Teófilo(a) Daniel Vilela Leonel(b) Teresa Lamano ${ }^{(c)}$}

(a) PhD, Assistant Professor; (c)Titular Professor Department of Morphology, Stomatology and Physiology; School of Dentistry of Ribeirão Preto, University of São Paulo, Ribeirão Preto, SP, Brazil.

(b) Undergraduate student, School of Dentistry of Ribeirão Preto, University of São Paulo, Ribeirão Preto, SP, Brazil.

\section{Corresponding author:}

Juliana Mazzonetto Teófilo

Departamento de Morfologia, Estomatologia e Fisiologia

Faculdade de Odontologia de Ribeirão Preto - USP

Avenida do Café, s/n

Ribeirão Preto - SP - Brazil

CEP: 14040-904

E-mail: teofilo@forp.usp.br

Received for publication on Jul 06, 2009 Accepted for publication on Oct 08, 2009

\begin{abstract}
Epidemiological studies have suggested that cola beverage consumption may affect bone metabolism and increase bone fracture risk. Experimental evidence linking cola beverage consumption to deleterious effects on bone is lacking. Herein, we investigated whether cola beverage consumption from weaning to early puberty delays the rate of reparative bone formation inside the socket of an extracted tooth in rats. Twenty male Wistar rats received cola beverage (cola group) or tap water (control group) ad libitum from the age of 23 days until tooth extraction at 42 days and euthanasia 2 and 3 weeks later. The neoformed bone volume inside the alveolar socket was estimated in semi-serial longitudinal sections using a quantitative differential point-counting method. Histological examination suggested a decrease in the osteogenic process within the tooth sockets of rats from both cola groups, which had thinner and sparser new bone trabeculae. Histometric data confirmed that alveolar bone healing was significantly delayed in cola-fed rats at three weeks after tooth extraction (ANOVA, $\mathrm{p}=0.0006$, followed by Tukey's test, $\mathrm{p}<0.01$ ). Although the results of studies in rats cannot be extrapolated directly to human clinical dentistry, the present study provides evidence that cola beverage consumption negatively affect maxillary bone formation.
\end{abstract}

Descriptors: Cola; Bone regeneration; Alveolar process.

\section{Introduction}

A number of risk factors may negatively affect bone metabolism and predispose to fracture, including the excessive consumption of carbonated beverages. ${ }^{1,2}$ Experimental evidence showing an association between cola beverage intake and bone metabolism disorders come primarily from worldwide epidemiological studies showing that increased cola beverage intake is associated with reduced bone mineral accrual, increased bone fracture risk at any age and an increased risk of osteoporosis later in life. For example, a study that combined medical stories with foodfrequency questionnaires from American adolescents revealed a strong association between cola beverage consumption and bone fractures in girls. ${ }^{3}$ A cross-sectional observational study carried out in Northern Ireland also showed an inverse relationship between the intake of carbonated soft drinks and bone mineral density in the heels of girls between the ages of 12 to 15 years. ${ }^{4} \mathrm{~A}$ study of a large Tasmanian cohort showed an association between cola beverage consumption and increased risk of 
wrist and forearm fracture in boys and girls between the ages of 9 and 16 years. ${ }^{5}$ In Denmark, a recent investigation of blood and urinary markers of calcium homeostasis and bone turnover in young men between the ages of 22 and 29 years confirmed that cola beverage intake may negatively impact bone metabolism. ${ }^{6}$ A Framingham Osteoporosis Study, a long-term study in the United States, revealed that cola intake was associated with low bone mineral density in women's hips. ${ }^{7}$

Experimental evidence linking cola beverage consumption to effects on bone is lacking. Acute ingestion of cola drinks for seven days causes plasma and urinary signs of disrupted calcium and phosphate metabolism, which are more severe in immature (30-day-old) than in adult (90-day-old) rats. ${ }^{8}$ Diminished femoral mineralization was observed in ovariectomized rats ${ }^{9}$ and in normal male and female rats ${ }^{10}$ submitted to prolonged ingestion of cola beverages.

Investigations carried out in humans ${ }^{7}$ and in animal models ${ }^{9}$ confirm that different bones respond in a distinct manner when exposed to adverse conditions, including cola beverage intake. To the best of our knowledge, none of the epidemiological or experimental studies to date have reported the effect of cola beverage consumption on alveolar bone, a bone region of major interest in dentistry. Filling of an extracted tooth socket by neoformed bone can be used experimentally to investigate the metabolism of alveolar bone under specific conditions. The present study is a histometric investigation of whether cola beverage consumption from weaning to early puberty delays alveolar bone formation in rats.

\section{Materials and Methods}

Twenty weaning 23-day-old male Wistar rats were housed in a climate-controlled room $(12 \mathrm{~h}$ light, $23-25^{\circ} \mathrm{C}$ ) with free access to laboratory chow plus cola beverage (cola groups) or water (control groups) (5 animals per plastic box measuring $40 \times 32 \times 17 \mathrm{~cm})$. Known amounts of solid food and liquid (tap water or cola beverage) were offered once a week and their consumption was estimated per cage during a $24 \mathrm{~h}$ period; the mean body weights of five animals in each cage were then calculated, and the amount of liquid and food ingested per $100 \mathrm{~g}$ of body weight were estimated.

At the age of 42 days the rats were anaesthetized by intraperitoneal injection of ketamine hydrochloride (União Química Farmacêutica Nacional S/A, Embu-Guaçu, SP, Brazil; $75 \mathrm{mg} / \mathrm{kg}$ ) and xylazine (Laboratórios Calier do Brasil Ltda., São Paulo, SP, Brazil; $10 \mathrm{mg} / \mathrm{kg}$ ) and the upper right incisors were extracted with a forceps after disconnecting the surrounding gingiva and luxation with the cutting edge of an enamel hatchet. After the tooth was extracted, the wounds were sutured with mononylon 4-0 (Ethicon, Johnson \& Johnson, São José dos Campos, SP, Brazil) and a single dose of antibiotic $(0.2 \mathrm{~mL} / \mathrm{rat}$, intramuscularly: Pentabiótico Veterinário, Wyeth, São Bernardo do Campo, SP, Brazil) was administered.

The animals were euthanized at either two or three weeks postoperatively (early puberty, $\mathrm{n}=5$ per group) with an intraperitoneal overdose of anesthetic. All procedures were conducted in compliance with ethical principles for animal research (Protocol 05.1.242.53.1).

Immediately after death, the heads were immersed in $10 \%$ formalin solution for $48 \mathrm{~h}$, and the maxillae were dissected away, decalcified (6 days in a solution containing $20 \%$ sodium citrate and $30 \%$ formic acid, replaced every 2 days) and processed for paraffin embedding. Semi-serial longitudinal 6$\mu \mathrm{m}$-thick sections of the alveolar sockets were cut at $60-\mu \mathrm{m}$ intervals and stained with hematoxylin and eosin.

The healing process, which consists of a gradual replacement of connective tissue by bone trabeculae in this phase, was estimated by new bone volume fraction (\% bone trabeculae relative to bone trabeculae plus connective tissue) using optical microscopy with a digital camera for image capture (Axio Star Plus and Axiovision; Zeiss; Germany) and a public domain histometry software (Image J, USA). A total of between 1,000-1,400 points were counted in 5-6 histological sections per alveolus (total area measured $5.2-6.3 \times 10^{3} \mu \mathrm{m}^{2}$, final magnification $100 \mathrm{X}$ ), with the percentage of points lying on bone trabeculae being proportional to their volume density. The measurements were made blindly by one investigator and were standardized in the apical alveolar third to avoid interference due to regional 
differences in the rate of bone healing.

Differences between the experimental and control groups were analyzed by one way ANOVA followed by Tukey's multiple comparison test after the D'Agostino and Pearson normality test showed a normal distribution of sample data (GraphPad Prism 4 software, $\mathrm{p}<0.05$ for statistical significance).

\section{Results}

Histological examination carried out in control rats two weeks after the tooth extraction showed the alveolar socket occupied by mature connective tissue and delicate bone trabeculae lined with osteoblasts (Figure 1A), which formed from the inner surfaces of the alveolar walls. Blood clot remnants were still observed, mainly in the central region. By the third week, most of the socket was filled with a network of thicker trabecular bone in control rats (Figure 1B). Relative to the respective controls, the osteogenic process appeared to be delayed in the socket of cola consuming rats at either two or three weeks after tooth extraction (Figures $1 \mathrm{C}, \mathrm{D}$ ), in which new bone trabeculae appeared thinner and sparser.

Histometric data confirmed the histological observation that cola beverage consumption was associated with a significant delay in alveolar bone formation (one way ANOVA, $\mathrm{p}=0.0006$ ). A $27.60 \%$ decrease in the mean percentage of new bone trabeculae was observed in cola beverage consuming rats three weeks after tooth extraction $(\mathrm{p}<0.01)$. Bone healing decreased by $25.75 \%$ in cola beverage consuming rats two weeks after tooth extraction,
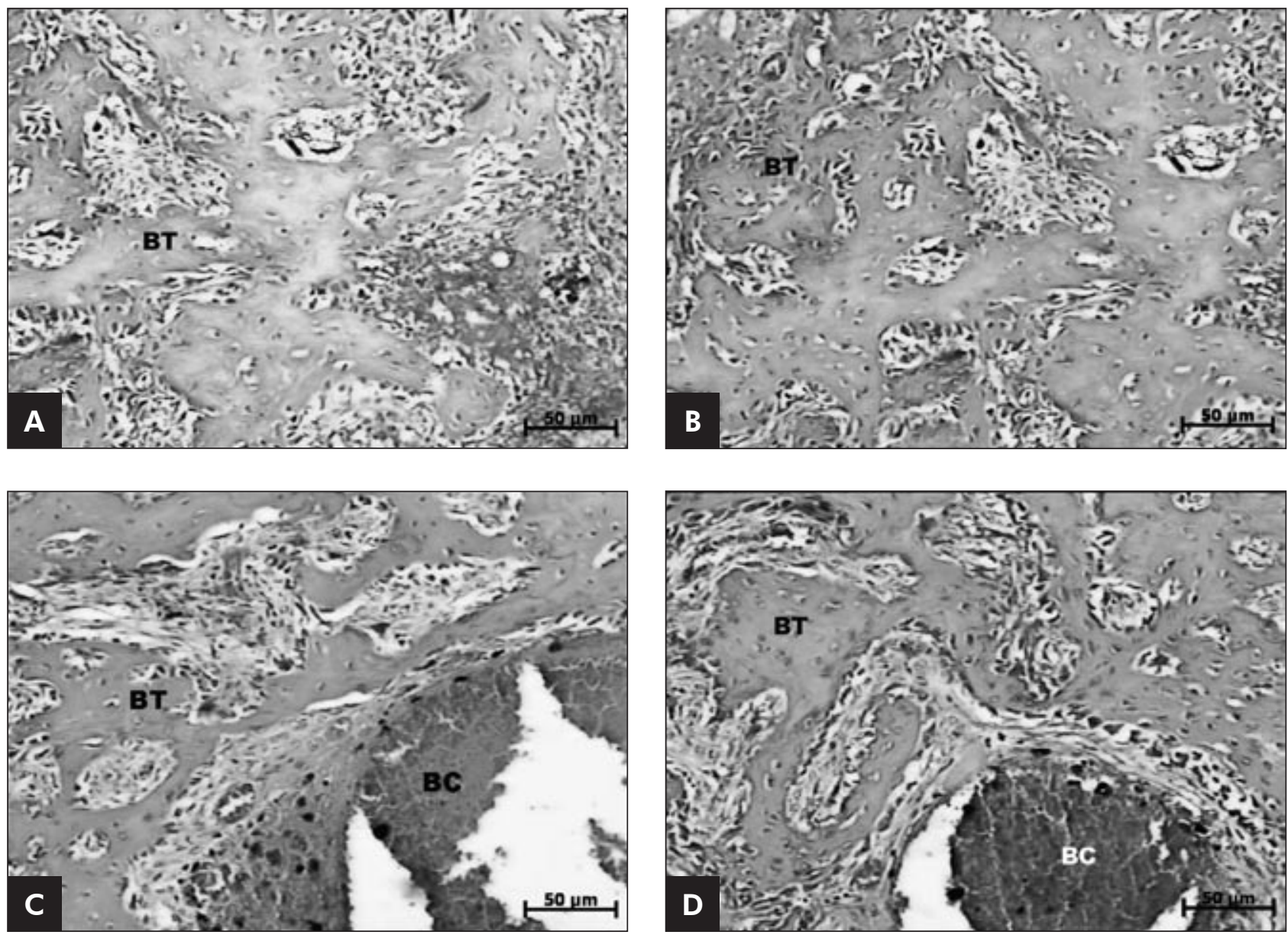

Figure 1 - Alveolar apical third of rats from the control groups at two (A) and three (B) weeks after tooth extraction and from the cola consuming groups at two (C) and three (D) weeks after tooth extraction. Note the neoformed bone trabeculae (BT) and blood clot remnants (BC) inside the alveolar socket (Hematoxylin and Eosin, $100 \mathrm{X}$ ). 
but the difference was not statistically significant $(\mathrm{p}>0.05)($ Graph 1).

\section{Discussion}

In the present study, the intra-alveolar bone healing in control rats closely resembled data in the literature. ${ }^{11}$ Briefly, after tooth extraction the socket fills with a blood clot, which is progressively invaded by capillary sprouting and fibroblasts originating from periodontal ligament remnants. During this process, the coagulum is gradually absorbed and replaced by immature connective tissue, the amount of inflammatory cell and blood vessels decreases and the osteoblasts become evident. The osteoblasts initially synthesize an immature bone matrix (osteoid) which is further mineralized by calcium deposition as hydroxyapatite crystals. The alveolar bone neoformation propagates from the apical and lateral walls towards the center and the healing process culminates with the filling of the dental socket by trabecular bone. ${ }^{11,12}$ Studies in rats have confirmed that maximal bone formation ${ }^{13}$ and maximal bone mineral density ${ }^{12}$ occur by the end of the second week after tooth extraction, the first period chosen for bone healing measurement in the present study.

Treated rats received cola beverage ad libitum from weaning ( 23 days old) until tooth extraction at prepuberty (42 days old) and the tooth sockets were examined either two or three weeks later, at early

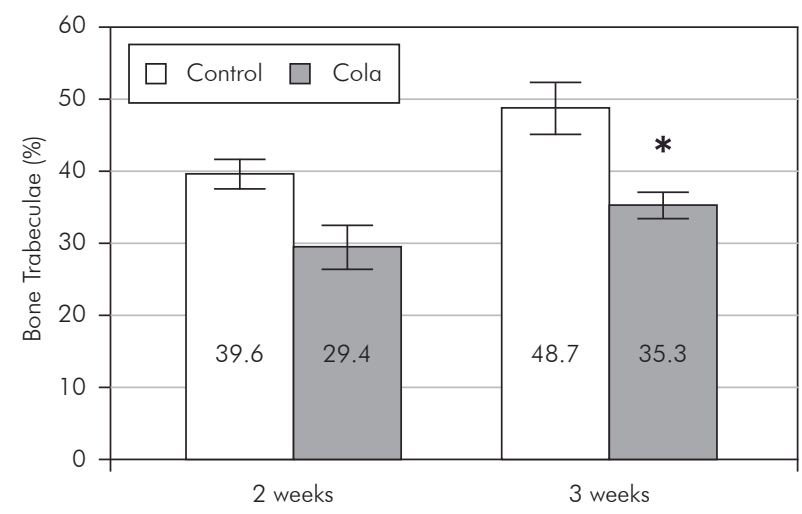

Graph 1 - Neoformed bone trabeculae (\%) (mean \pm SEM) in the alveolar apical third of rats from the control and cola groups at two and three weeks after tooth extraction (one way ANOVA followed by Tukey's multiple comparison test, *statistically significant difference between the cola group and respective control, $p<0.01$ ). puberty - the phases of development were defined according to Zanato et al. ${ }^{14}$ (1994). It is worth mentioning that cola beverage ingestion did not interfere perceptibly with the amount of solid food ingested and did not change body weight gain during the experimental period; it is also likely that fluid intake did not differ significantly between animals in the control and cola beverage consuming groups (data not shown).

Histological examination, confirmed by histometric data, showed a significant delay in alveolar bone formation in cola consuming rats at two or three weeks after tooth extraction.

The molecular mechanisms that underlie the disruption of bone metabolism by cola beverage consumption remain unclear. Epidemiological studies of the trend toward replacing milk, a major calcium source, with soft drinks suggest that poor nutrition may be a risk factor that predisposes to bone fracture. ${ }^{1,6}$ In contrast, other reports suggest that decreased milk intake is not associated with increased fracture risk in children ${ }^{5}$ and low bone mineral density in adult women. ${ }^{7}$ In agreement with these earlier reports, pair-fed rats consuming tap water did not develop signs of bone disorder (hypocalcemia and reduced femoral mineralization) which were observed in rats that ingested high levels of cola drinks. ${ }^{9}$

The association between bone metabolism disorders and cola beverage intake has also been attributed to some components of the cola beverage formulation, some of which is confidential with regard to constituents and quantities: the exact composition of the cola beverage syrup is an industrial trade secret.

Among the potentially deleterious constituents, phosphoric acid can reduce 25-dihydroxyvitaminD synthesis, interfere with intestinal absorption and interfere with renal reabsorption of calcium leading to hypocalcemia and causing secondary hyperparathyroidism which appears insufficient to revert to a low calcium plasma level. ${ }^{9,15}$

Furthermore, cola beverages are rich in caffeine, which has been associated with reduced bone mineral density and increased fracture risk. ${ }^{16,17}$ According to Kinney ${ }^{18}$ (2002), non-cola carbonated soft drinks, which do not contain phosphoric acid or caffeine, have not been associated with fracture risk among 
children and adolescents. In contrast, a recent epidemiological study suggested that caffeine cannot explain the reduction in bone mineral density in spine of women who ingest cola beverages, since both caffeinated and decaffeinated cola drinks produced similar degrees of bone disturbances. ${ }^{7}$

It is known that acidifying dietary constituents may also negatively affect calcium metabolism and accelerate bone resorption. ${ }^{19}$ Thus, another possible mechanism underlying the effect on bone metabolism may be the acid load characteristic of cola drinks, which may adversely affect calcium and bone metabolism by increasing bone resorption and

\section{References}

1. Goulding A. Risk factors for fractures in normally active children and adolescents. Med Sport Sci. 2007;51:102-20.

2. Root AW. Bone strength and the adolescent. Adolesc Med. 2002 Feb;13(1):53-72.

3. Wyshak G, Frisch RF. Carbonated beverages, dietary calcium, the dietary calcium/phosphorus ratio, and bone fractures in girls and boys. J Adolesc Health. 1994 May;15(3):210-5.

4. McGartland C, Robson PJ, Murray L, Cran G, Savage MJ, Watkins D, et al. Carbonated soft drink consumption and bone mineral density in adolescence: the Northern Ireland young hearts project. J Bone Miner Res. 2003 Sep;18(9):1563-9.

5. Ma D, Jones G. Soft drink and milk consumption, physical activity, bone mass, and upper limb fractures in children: a population-based case-control study. Calcif Tissue Int. 2004 Oct;75(4):286-91.

6. Kristensen M, Jensen M, Kudsk J, Henriksen M, Molgaard C. Short-term effects on bone turnover of replacing milk with cola beverages: a 10-day interventional study in young men. Osteoporos Int. 2005 Dec;16(12):1803-8.

7. Tucker KL, Morita K, Qiao N, Hannan MT, Cupples LA, Kiel D. Colas, but not other carbonated beverages, are associated with low bone mineral density in older women: The Framinghan Osteoporosis Study. Am J Clin Nutr. 2006 Oct;84(4):93642.

8. Amato D, Maravilla A, Montova C, Gaja O, Revilla C, Guerra $\mathrm{R}$, et al. Acute effects of soft drink intake on calcium and phosphate metabolism in immature and adult rats. Rev Invest Clin. 1998 May-Jun;50(3):185-9.

9. Garcia-Contreras F, Paniagua R, Avila-Díaz M, CabreraMunõz L, Martinez-Muniz I, Foyo-Niembro E, et al. Cola beverage consumption induces bone mineralization reduction in ovariectomized rats. Arch Med Res. 2000 Jul-Aug;31(4):3605.

10. Ogur R, Uysal B, Ogur T, Yaman H, Oztas E, Ozdemir A, et al. Evaluation of the effect of cola drinks on bone mineral calcium mobilization and reducing the renal production of 25-hydroxy-vitamin-D. ${ }^{9}$

\section{Conclusion}

In conclusion, ad libitum ingestion of cola beverage from weaning until early puberty disrupted maxillary bone formation in rats, as confirmed by a significant delay in alveolar bone healing.

\section{Acknowledgements}

The authors are grateful for the financial support by FAPESP (Process 06/58184-6) and CNPq (Process 2007.1.389.58.5)

density and associated factors. Basic Clin Pharmacol Toxicol. 2007 May;100(5):334-8.

11. Lamano Carvalho TL, Bombonato KF, Brentegani LG. Histometric analysis of rat alveolar wound healing. Braz Dent J. 1997;8(1):9-12.

12. Elsubeihi ES, Heersche JN. Quantitative assessment of post-extraction healing and alveolar ridge remodelling of the mandible in female rats. Arch Oral Biol. 2004 May;49(5):401-12.

13. Guglielmotti MB, Cabrini RL. Alveolar wound healing and ridge remodeling after tooth extraction in the rat: a histologic, radiographic and histometric study. J Oral Maxillofac Surg. 1985 May;43(5):359-64.

14. Zanato VF, Martins MP, Anselmo-Franci JA, Petenusci SO, Lamano Carvalho TL. Sexual development of male Wistar rats. Braz J Med Biol Res. 1994 May;27(5):1273-80.

15. Guerrero-Romero F, Rodriguez-Moran M, Reyes E. Consumption of soft drinks with phosphoric acid as a risk factor for the development of hypocalcemia in postmenopausal women. J Clin Epidemiol. 1999 Oct;52(10):1007-10.

16. Hernández-Avila M, Stampfer MJ, Ravnikar VA, Willet WC, Schiff I, Francis M, et al.. Caffeine and other predictors of bone density among pre and perimenopausal women. Epidemiology. 1993 Mar;4(6):128-34.

17. Ohta M, Cheuk G, Thomas KA, Kamagata-Kiyoura Y, Wonk CS, Yazdani M, et al. Effect of caffeine on the bones of aged, ovariectomized rats. Ann Nutr Metab. 1999 Jan-Feb;43(1):529.

18. Kinney MA. Does consumption of cola beverages cause bone fractures in children? Mayo Clin Proc. 2002 Sep;77(9):10056.

19. Buclin T, Cosma M, Appenzeller, Jacquet AF, Décosterd LA, Biollaz J, et al. Diet acids and alkalis influence calcium retention in bone. Osteoporos Int. 2001 Jun;12(6):493-9. 